\title{
IDH1 mutation diminishes aggressive phenotype in glioma stem cells
}

\author{
QI YAO $^{1 *}$, GANG CAI $^{2 *}$, QI YU ${ }^{2}$, JIANHONG SHEN $^{1}$, ZHIKAI GU $^{1}$, \\ JIAN CHEN $^{1}$, WEI SHI ${ }^{1}$ and JINLONG SHI ${ }^{1}$ \\ ${ }^{1}$ Department of Neurosurgery, The Affiliated Hospital of Nantong University, Nantong, Jiangsu 226001; \\ ${ }^{2}$ Department of Neurosurgery, The First People's Hospital of Nantong, Nantong, Jiangsu 226001, P.R. China
}

Received July 10, 2017; Accepted October 20, 2017

DOI: 10.3892/ijo.2017.4186

\begin{abstract}
The R132H mutation in isocitrate dehydrogenase 1 (IDH1-R132H) is associated with better prognosis in glioma patients. Glioma stem cells (GSCs) in glioma are believed to be responsible for glioma growth and maintenance. However, the relation between the $\mathrm{R} 132 \mathrm{H}$ mutation and GSCs is not fully understood. In the present study, GSC markers were detected in patients with IDH1-R132H or wild-type IDH1 (IDH1-wt) by tissue microarray immunohistochemistry (TMA-IHC). The relationship between the expression patterns of GSC markers and the clinicopathological characteristics in glioma were analyzed. To confirm this mutation's role in GSCs, the IDH1$\mathrm{R} 132 \mathrm{H}$ in GSCs isolated from glioblastoma patients with IDH1 mutations was overexpressed by using lentiviral constructs in vitro, and then the proliferation, differentiation, apoptosis, migration and invasion of the transfected GSCs were explored. At the molecular level, we detected $\mathrm{Wnt} / \beta$-catenin signaling expression to verify its role in regulating the cellular properties of GSCs. The results showed that the positive rate of GSCs in patients with IDH1-R132H was significantly less than that in patients with IDH1-wt. The positive rate of GSCs was correlated with IDH1 mutation, TNM stage and poor overall survive. After transfection in vitro, IDH1-R132H overexpression led to reduced GSCs proliferation, migration and invasion, inducing apoptosis and improving GSC differentiation, accompanied by a significant reduction in activity of $\beta$-catenin. Several mediators, effectors and targets of the $\mathrm{Wnt} / \beta$-catenin signaling were downregulated. The data demonstrate that IDH1 mutation reduces the malignant progression of glioma by causing a less aggressive phenotype of GSCs which are involved in the Wnt/ $\beta$-catenin signaling.
\end{abstract}

Correspondence to: Professor Wei Shi or Professor Jinlong Shi, Department of Neurosurgery, The Affiliated Hospital of Nantong University, 20 Xisi Road, Nantong, Jiangsu 226001, P.R. China

E-mail: ntshiwei123@163.com

E-mail: jinlong.shi@hotmail.com

*Contributed equally

Key words: glioma, glioma stem cells, isocitrate dehydrogenase 1, mutation, $\beta$-catenin

\section{Introduction}

Glioma is the most common primary intraparenchymal brain tumor in the central nervous system (CNS), accounting for approximately $7 \%$ of the death caused by cancer $(1,2)$. It is divided into grade I-IV with increasing severity by the World Health Organization (WHO). Although significant improvements in treatments for glioma, highly invasive WHO high-grade glioma exhibits a relentless malignant progression, resistance to all therapeutic approaches and ultimately leads to the patient's death (3). There is an urgent need for further research on the pathogenesis of glioma and improve the outcome of patients.

As has been shown in many tumors, glioma stem cells (GSCs), a small subpopulation of stem cells with enhanced self-renewal capacity and a multilineage differentiation potential, are believed to be the real driving force for tumor initiation, progression and relapse (4-6). GSCs give rise to a variety of proliferating and differentiated cells that make up the tumor mass and play a key role in resistance to radiotherapy and chemotherapy (7,8), angiogenesis (9) and metastasis (10). A recent study showed that temozolomide (a mainstay of chemotherapy in high-grade glioma) actually increased the proportion of GSCs by inducing conversion of non-GSCs into GSCs (4). Thus, the biology of GSCs should be explored in order to effectively enhance the treatment in glioma (11).

Investigation of genetic mutations associated with glioma showed that patients frequently carry isocitrate dehydrogenase 1 (IDH1) mutation (12-14). IDH1 are frequently mutated in WHO grade II and grade III glioma and in secondary glioblastoma. Approximately $90 \%$ of the IDH1 mutation in glioma is $\mathrm{R} 132 \mathrm{H}(15,16)$. IDH1 mutation results in production and accumulation of the metabolite D-2-hydroxyglutarate (D-2-HG) in IDH1 mutated cells, which greatly impacts the cellular metabolism and contributes to tumor pathogenesis $(13,17,18)$. IDH1 mutation is expected to be stable and homogenous among all tumor cells within IDH1-mutated glioma. As a genetic mutation, it is supposed to occur at the tumor precursor cell stage (19). However, the full effects of mutant IDH1 on GSCs biology are not fully understood. Our previous study suggested that the R132H mutation in IDH1 serves a tumor suppressor function in human glioma cells by negatively regulating Wnt/ $\beta$-catenin signaling (20). The vital role of the 
Table I. GSCs marker and clinicopathological parameters in 172 glioma specimens.

\begin{tabular}{|c|c|c|c|c|c|c|}
\hline \multirow[b]{2}{*}{ Variable } & \multirow[b]{2}{*}{ Total } & \multicolumn{2}{|c|}{ CD133 and nestin } & \multirow[b]{2}{*}{$\chi^{2}$} & \multirow[b]{2}{*}{ P-value } & \multirow[b]{2}{*}{$\mathrm{r}$} \\
\hline & & Positive & Negative & & & \\
\hline Age (years) & & & & 3.276 & 0.070 & -0.138 \\
\hline$<40$ & 32 & 12 & 20 & & & \\
\hline$\geq 40$ & 140 & 31 & 109 & & & \\
\hline Sex & & & & 0.303 & 0.582 & -0.042 \\
\hline Male & 110 & 29 & 81 & & & \\
\hline Female & 62 & 14 & 48 & & & \\
\hline Tumor location & & & & 4.067 & 0.397 & 0.035 \\
\hline Frontal & 74 & 15 & 59 & & & \\
\hline Parietal & 15 & 6 & 9 & & & \\
\hline Occipital & 26 & 7 & 19 & & & \\
\hline Temporal & 31 & 10 & 21 & & & \\
\hline Unknown & 26 & 5 & 21 & & & \\
\hline Surgery & & & & 0.062 & 0.969 & -0.009 \\
\hline Biopsy & 24 & 6 & 18 & & & \\
\hline Partial resection & 26 & 7 & 19 & & & \\
\hline Gross total resection & 122 & 30 & 92 & & & \\
\hline Tumor diameter $(\mathrm{cm})$ & & & & 2.005 & 0.157 & -0.108 \\
\hline$<4$ & 117 & 33 & 84 & & & \\
\hline$\geq 4$ & 55 & 10 & 45 & & & \\
\hline \multicolumn{7}{|l|}{ IDH1-mu } \\
\hline Positive & 48 & 6 & 42 & 5.548 & 0.018 & 0.180 \\
\hline Negative & 124 & 37 & 87 & & & \\
\hline TNM grade & & & & 17.128 & 0.001 & 0.274 \\
\hline I & 15 & 3 & 12 & & & \\
\hline II & 54 & 4 & 50 & & & \\
\hline III & 53 & 15 & 38 & & & \\
\hline IV & 50 & 21 & 29 & & & \\
\hline
\end{tabular}

Wnt signaling pathway for the function of normal and cancer stem cells is commonly accepted (21). One of the hallmarks of stem cells is the ability to maintain long telomeres by function of the TERT gene. TERT expression is directly enhanced by binding of $\beta$-catenin to its promoter region and thereby links telomerase activity to Wnt signaling (22). In the present study, we investigated the role of IDH1-R132H in GSCs and detected $\mathrm{Wnt} / \beta$-catenin signaling expression to verify its role in regulating the cellular properties of GSCs.

\section{Materials and methods}

Clinical samples and cell line. The samples used in the present study were obtained from 172 patients newly diagnosed with glioma that underwent sample resection without therapy between 2010 and 2015 at the Department of Neurological Surgery, the Affiliated Hospital of Nantong University. The collection of the glioma specimens was performed in accor- dance with the protocols approved by the Ethics Committee of the Affiliated Hospital of Nantong University. The clinical demographic details of the 172 patients are outlined in Table I. Specimens were snapped frozen in liquid nitrogen immediately after surgery and stored at $-80^{\circ} \mathrm{C}$ until use.

Human GSCs used in the present study were isolated from glioblastoma patients with IDH1 mutation as previously described $(23,24)$. In brief, a few minutes after tumor removal, tissues were dissociated into a cell suspension by $0.25 \%$ trypsin digestion. Cells that passed through a $100 \mu \mathrm{m}$ strainer (Falcon, Oxnard, CA, USA) were cultured in the medium composed of Dulbecco's modified Eagle's medium (DMEM) with 2\% B27, epidermal growth factor (EGF, $10 \mathrm{ng} / \mathrm{ml}$ ) and basic fibroblast growth factor (bFGF, $10 \mathrm{ng} / \mathrm{ml}$ ). Cells were incubated at $37^{\circ} \mathrm{C}$ with $95 \%$ air, $5 \% \mathrm{CO}_{2}$ and $100 \%$ humidity. Two weeks later, floating primary tumor spheres were collected. The sphere cells were harvested, dissociated into single cells and plated into a 24-well plate for the subsphere-forming. Subspheres 
from one mother cell were characterized by immunocytochemistry against CD133 and nestin.

To induce differentiation of GSCs, the cells $\left(1 \times 10^{5}\right.$ cells $\left./ \mathrm{ml}\right)$ were plated onto poly-D-lysine coated coverslips in 96-well plates containing DMEM with $10 \%$ fetal bovine serum (FBS).

Tissue microarray immunohistochemistry (TMA-IHC). Immunohistochemistry was performed using protocols described previously by Gurung et al (25). In brief, the TMA slides were deparaffinised, rehydrated, washed and endogenous peroxidase was blocked using Bond-III 'Dewax Protocol D' following the manufacturer's instructions (Leica Biosystems, Newcastle, UK). Epitope retrieval was achieved using Bond-III 'Protocol H1(30)' (Leica Biosystems). The slides were incubated with antibodies against anti-IDH1R132H (1:400; Dianova GmbH, Hamburg, Germany), CD133 (1:500; Abcam, Cambridge, UK) or nestin (1:500; Abcam) at room temperature (RT) for $1 \mathrm{~h}$. Antibody binding was detected using diaminobenzidine (DAB) with hematoxylin counterstaining following Bond-max and Bond-x 'IHC protocol F' (Leica Biosystems). The immunostaining results were interpreted independently by two expert pathologists who were blinded to the clinical data. The staining intensity was scored using the following scale of four grades: 0 , no staining; 1, weak staining; 2, moderate staining; and 3, strong staining. At least 5 areas of each core were viewed and the proportion of cells in each core staining positively was assigned a score (0-100\%). A semi-quantitative histopathology $(\mathrm{H})$ score was obtained by multiplying the staining intensity score with the percentage score (0-300). An H-score higher than the median was considered positive.

Generation of lentiviral constructs and transfection. To overexpress IDH1-wt, the coding sequence of IDH1 was amplified using the following primers: forward primer 5'-ATGTCCAAA AAAATCAGTGGCGG-3' and reverse primer 5'-GTTTGGCC TGAGCTAGTTTG-3'. This fragment was then sub cloned into the lentiviral vector pLenti6.3-MCS-IRES2-EGFP vector (Invitrogen, Carlsbad, CA, USA) using the restriction sites for BamHI and AscI. To overexpress the R132H mutant form of IDH1, we procured commercial synthetic sequences of IDH1 mutated at Arg 132, and sub-cloned them into the pLenti6.3MCS-IRES2-EGFP vector. An empty vector (EV) was used as the control. The vectors and the Packaging Mix were cotransfected into the 293FT cell line to generate lentiviral stocks, which were used to transfect the target cells.

Immunofluorescent staining. For immunofluorescent staining, cells were first fixed in 4\% PFA, followed by incubation in hydrogen peroxide, blocking of non-specific antibody binding sites, and overnight incubation with the following primary antibodieså: rat anti- $\beta$-catenin $(1: 2,000 ;$ Abcam), mouse monoclonal anti-nestin (1:600; Roche Diagnostics GmbH, Mannheim, Germany), rat anti-CD133 (1:600; Roche Diagnostics $\mathrm{GmbH}$ ) and mouse monoclonal anti-MAP-2 (1:600; Roche Diagnostics $\mathrm{GmbH}$ ). The following day, cells were labeled secondary antibody with Alexa Fluor 568-conjugated goat anti-rat (1:1,000; Molecular Probes, Eugene, OR, USA) or 488-conjugated goat anti-mouse (1:800; Invitrogen). Cell nuclei were counter-stained with Hoechst 33342 for
30 min at RT. Immunopositive cells were observed under a fluorescent microscope.

Real-time PCR analysis. To determine the level of mRNA expression in cells, the total RNA was extracted from $1 \times 10^{6}$ cells in each group using UNIQ-10 Spin Column RNA Purified kit (Sangon Biotech Co., Ltd., Shanghai, China). The first strand cDNA was synthesized using RevertAid ${ }^{\mathrm{TM}}$ First Strand cDNA Synthesized kit (Fermentas, Burlington, ON, Canada). First Strand cDNA was subsequently subjected to Corbett RG-6000 PCR system (Qiagen, Dusseldorf, German) using FastStart Universal SYBR-Green Master Mix (Roche, Basel, Switzerland). The reactions were optimized by varying the annealing temperatures from 50 to $55^{\circ} \mathrm{C}$, the sense and antisense primers were synthesized as follows: GAPDH 5'-GCAA GTTCAACGGCACAG-3' and 5'-GCCAGTAGACTCCACG ACAT-3'; CD133 5'-TTCTGCCTGTGTAACTTTGCA-3' and R, 5'-TTGTTGTGCAACGTCTTCAAGTAT-3'; nestin 5'-AG CGTTGGAACAGAGGTTGGA-3' and 5'-TGTTTCCTCCCA CCCTGTGTCT-3'; $\beta$-catenin 5'-ATTGAAGCTGAGGGAGC CAC-3' and 5'-TCCTGGCCATATCCACCAGA-3'; LEF1 5'-AAATAAAGTGCCCGTGGTGC-3' and 5'-CATGCCTTG TTTGGAGTTGACA-3'; TCF4 5'-CGGCGGTGGGGGGA TGAC-3' and 5'-GGCCGCTTCTTCCAAACTTTCC-3'.

Western blot analysis. To determine the level of protein expression in cells, aliquots of total protein (50 $\mu \mathrm{g}$ per lane) were electrophoresed on a $12 \%$ SDS-polyacrylamide gradient gel and transferred to nitrocellulose membranes (Millipore, Darmstadt, Germany). Washed in rinse buffer at RT and incubated in blocking buffer (5\% fat-free milk in rinse buffer) for $30 \mathrm{~min}$, the membranes were incubated for $2 \mathrm{~h}$ at RT with the following primary antibodies: anti-IDH1 R132H (1:500; Abcam;), anti-IDH1 (1:500; Dianova GmbH), anti$\beta$-catenin (1:4,000; Abcam), anti-LEF1 (1:500; Santa Cruz Biotechnolgy, Santa Cruz, CA, USA), anti-TCF4 (1:200; Santa Cruz Biotechnolgy), anti-nestin (1:600; Roche Diagnostics $\mathrm{GmbH}$ ), anti-CD133 (1:600; Roche Diagnostics GmbH) and anti-MAP-2 (1:800; Invitrogen). The following day, they were incubated with the HRP-conjugated secondary antibodies and visualized using the enhanced chemiluminescence (ECL) system (Pierce Biotechnology Inc., Rockford, IL, USA). In addition, $\beta$-actin was used as a reference protein.

Proliferation and apoptosis assay of GSCs. The cells of EV, IDH1-wt and IDH1-mu groups were cultured in the above mentioned medium. At day 7 in vitro (DIV), some cell spheres of three groups were dissociated into single-cell suspension mechanically by trypsin digestion. Collected cells were washed once in cold phosphate-buffered saline (PBS). Cells were analyzed using Cell Cycle and Apoptosis Analysis kit (Beyotime Institute of Biotechnology, Haimen, China) by flow cytometry (Epics XL; Beckman Coulter, Fullerton CA, USA), according to the manufacturer's protocol.

Invasion and migration assays of GSCs. To measure changes in cell migration, we performed the Transwell migration assay using 24-well BD Matrigel invasion chambers (BD Biosciences, San Jose, CA, USA) as previously described (20). In brief, $5 \times 10^{4}$ cells were seeded into the upper well in DMEM 
(without serum) and incubated for $24 \mathrm{~h}$ to allow the cells to migrate into the lower well through an $8-\mu \mathrm{m}$ pore membrane. After $24 \mathrm{~h}$, the latter were fixed and stained for easy visualization and quantification. The average number of cells per field was determined by counting the number of cells in six random fields per well. To determine the invasive properties, cells were plated into chambers coated with Matrigel, which were then inserted into a 24-well plate and incubated for $48 \mathrm{~h}$ in DMEM and $10 \%$ FBS. At the end of the assay, the cells that adhered to the lower well were fixed, stained and visualized.

Statistical analysis. Statistical analysis was performed using statistics package for social science 21.0 (SPSS 21.0). The group comparison was analyzed using the independent t-test or one-way ANOVA. Associations between positive rate of GSC markers and the clinicopathological characteristics were analyzed using the Pearson's chi-squared test and the correlation analysis was compared using the Pearson test. Five-year overall survival (OS) was estimated using the Kaplan-Meier method and the differences in survival among groups were compared using the log-rank test. $\mathrm{P}<0.05$ was considered statistically significant.

\section{Results}

The number of GSCs was decreased in patients with IDH1R132H-mu and correlated with TNM stage and poor overall survival. The results of TMA-IHC showed that there were 48 patients with IDH1-mu and 124 patients with IDH1-wt. GSCs in patients with IDH1-mu or IDH1-wt were identified by CD133 and nestin TMA-IHC. Many CD133 ${ }^{+}$and nestin ${ }^{+}$cells were found in the patients with IDH1-wt while a few CD133+ and nestin ${ }^{+}$cells in the patients with IDH1-mu. The positive rate of GSCs in the patients with IDH1-wt was more than that in the patients with IDH1-mu. The difference among them was statistically significant $(\mathrm{P}<0.05)$ (Fig. 1A). The correlation between positive rate of GSC markers and a series of clinicopathological characteristics was conducted. However, the relationship between the expression patterns of GSC markers and the histologic grade in glioma was not analyzed in the present study because of the limited samples of high histologic grade glioma. In the following study, we will collect enough samples to carry out this analysis. As shown in Table I, positive rate of GSC marker was significantly correlated with tumor stage $(r=0.274)$ and IDH1 mutation $(r=0.180)$. Five-year OS of patients was estimated using the Kaplan-Meier. The median 5-year OS of patients with GSC marker negative was 41 months (95\% CI, 36.64-44.39 months). The median 5-year OS of patients with GSCs marker positive was 26 months $(95 \%$ CI, 19.28-31.89 months). The 5-year OS rate was significantly lower for GSC marker positive patients than for GSCs marker negative patients, the difference was statistically significant $(\mathrm{P}<0.05)$ (Fig. 1B).

The expression of IDH1-R132H in transfected GSCs in vitro. GSCs were identified by CD133 and nestin markers in vitro. The cells were cultured in serum-free medium, many cells formed floating primary tumor spheres. Subspheres from one mother cell were CD133 and nestin double positive (Fig. 2A). The expression of IDH1-R132H in GSCs was detected by
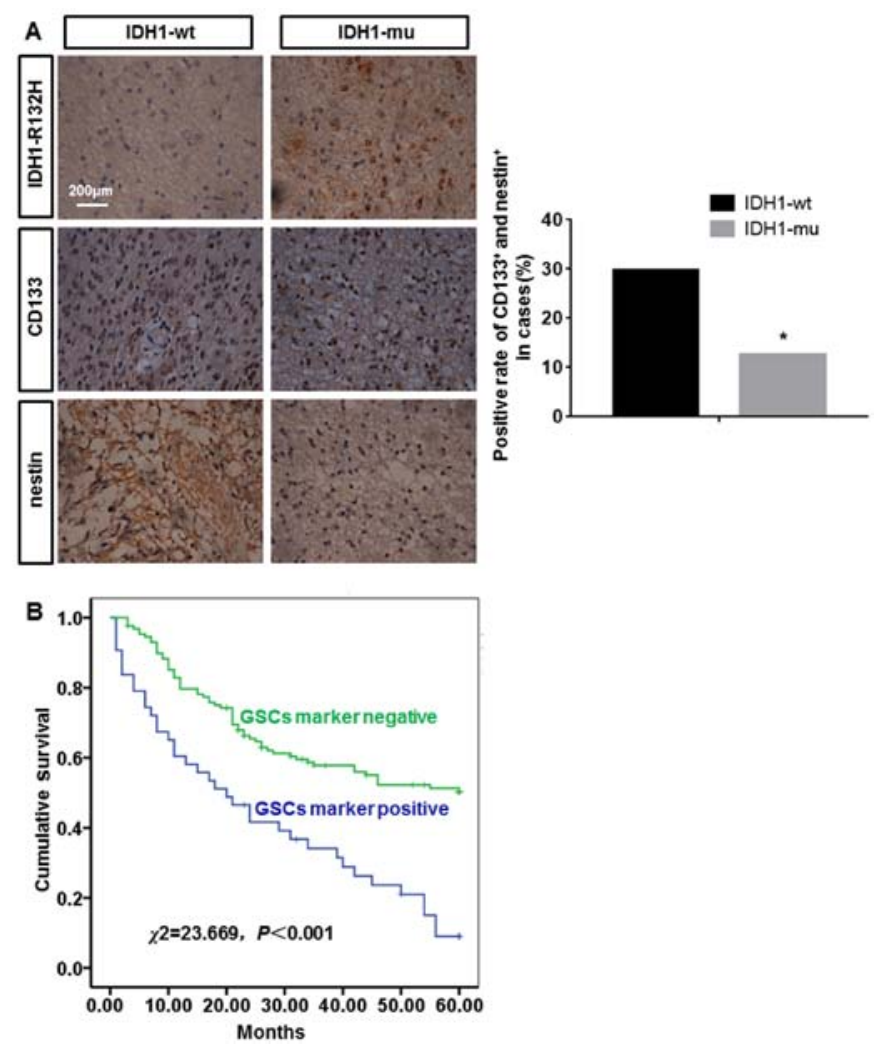

Figure 1. GSCs in human glioma tissue were identified by CD133 and nestin immunochemistry. (A) Many CD133 ${ }^{+}$and nestin ${ }^{+}$cells were found in the patients with IDH1-wt while a few $\mathrm{CD}_{133^{+}}$and nestin ${ }^{+}$cells in the patients with IDH1-R132H. Bar, $200 \mu \mathrm{m}$. The positive rate of GSCs in the patients with IDH1-wt was more than that in the patients with IDH1-mu. The difference between them was statistically significant. ${ }^{*} \mathrm{P}<0.05$ vs. IDH1-wt. (B) Five-year OS of patients was estimated using the Kaplan-Meier curves. The 5-year OS rate was significantly lower for GSCs marker positive patients than for GSCs marker negative patients, the difference was statistically significant $(\mathrm{P}<0.05)$

western blot analysis. IDH1-R132H protein level in IDH1$\mathrm{R} 132 \mathrm{H}$ modified GSCs was more than that in the EV and IDH1-wt modified GSCs. The difference among them was statistically significant $(\mathrm{P}<0.05)$ (Fig. 2B). The expression of CD33 or nestin mRNA and protein in the transfected GSCs were detected by RT-PCR and western blot analysis. CD33 or nestin mRNA and protein level in IDH1-R132H modified GSCs was lower than that in the EV and IDH1-wt modified GSCs $(\mathrm{P}<0.05)$ (Fig. 2C and D).

IDH1-R132H mutation reduced the activity of Wnt/ $\beta$-catenin signaling in GSCs. The $\beta$-catenin expression level was detected by RT-PCR, western blot analysis and immunofluorescence. Compared with IDH1-wt and EV groups, the mRNA and protein expression of $\beta$-catenin in IDH1-mu group were significantly decreased. The difference among them was statistically significant $(\mathrm{P}<0.05)$ (Fig. 3A). Many intense $\beta$-catenin ${ }^{+}$cells were found in the EV and IDH1-wt groups, and a few weak $\beta$-catenin ${ }^{+}$cells were found in the IDH1-mu group. The difference among them was statistically significant $(\mathrm{P}<0.05)$ (Fig. 3B). To further analyze the level of Wnt $/ \beta$-catenin signaling in the GSCs, the concentration of the $\beta$-catenin co-actors and Wnt signaling mediators TCF4 and 
A
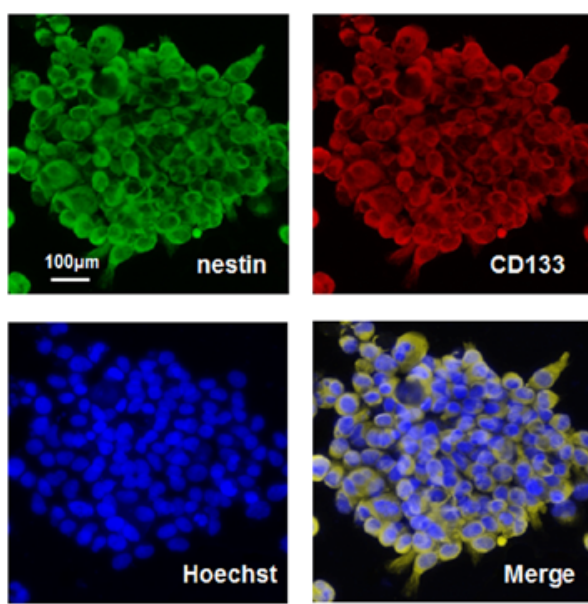

C

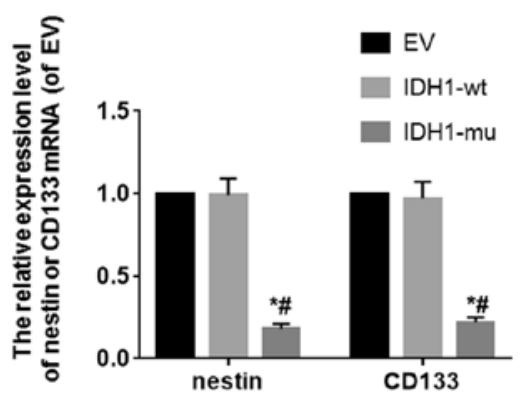

B
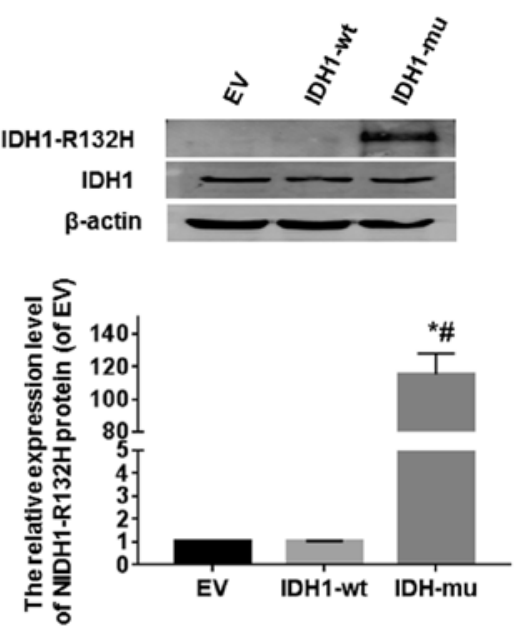

D

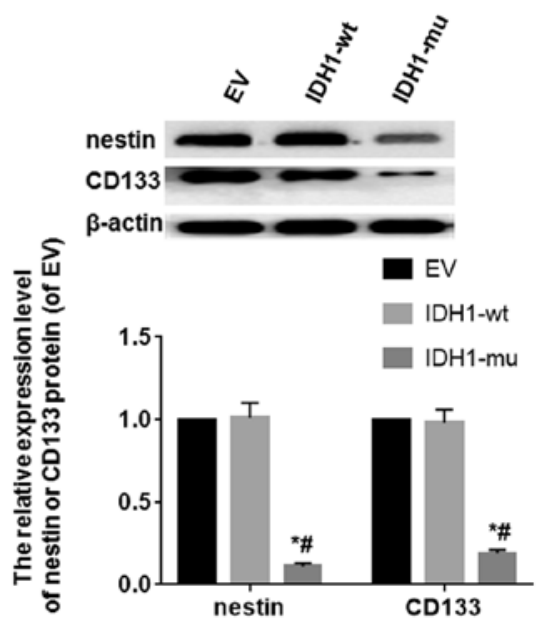

Figure 2. The expression of IDH1-R132H in transfected GSCs in vitro. (A) The GSCs were identified by CD133 and nestin markers. Subspheres from one mother cell were CD133 and nestin double positive. Bar, $100 \mu \mathrm{m}$. (B) After transfection, the expression of IDH1-R132H in GSCs was detected by western blot analysis. IDH1-R132H protein level in IDH1-mu group was more than that in the EV and IDH1-wt groups. (C) The expression of CD33 or nestin mRNA in the transfected GSCs was detected by RT-PCR. CD33 or nestin mRNA level in IDH1-R132H modified GSCs was lower than that in the EV and IDH1-wt modified GSCs. (D) The expression of CD33 or nestin protein in the transfected GSCs was detected by western blot analysis. CD33 or nestin protein level in IDH1-R132H modified GSCs was lower than that in the EV and IDH1-wt modified GSCs. ${ }^{*} \mathrm{P}<0.05$ vs. EV; ${ }^{\text {P }}<0.05$ vs. IDH1-wt.

LEF1 was measured by RT-PCR and western blot analysis. The mRNA and protein expression levels of TCF4 and LEF1 were downregulated in IDH1-mu group, compared with IDH1-wt and EV groups. The difference among them was statistically significant $(\mathrm{P}<0.05)$ (Fig. 3C).

IDH1-R132H mutation led to a less aggressive phenotype in GSCs. The proliferation ability of GSCs in 3 groups was detected by flow cytometry. The result showed the proliferation index (PI) of IDH1-mu group was 30.05 \pm 9.01 , while the PI of EV and IDH1-wt groups were 43.86 \pm 9.85 and 42.64 \pm 10.43 , respectively. The PI of IDH1-mu group was lower than that of other two groups $(\mathrm{P}<0.05)$ (Fig. 4A). The differentiation ability of GSCs in 3 groups was detected by immunofluorescence and western blot analysis. Scarce MAP $-2^{+}$neurons and many $\mathrm{CD}_{133^{+}}$cells were found in the 3 groups. Compared with IDH1-wt and EV group, the percentage of MAP-2 ${ }^{+}$neurons in IDH1-mu group was more than that of the other two groups, but the percentage of $\mathrm{CD}_{133^{+}}$cells in IDH1-mu group was less than that of the other two groups $(\mathrm{P}<0.05)$ (Fig. 4B). Compared with IDH1-wt and EV groups, the CD133 protein level was decreased and MAP-2 protein level was increased in the IDH1-mu group $(\mathrm{P}<0.05)$ (Fig. 4C). The apoptotic level of GSCs in 3 groups was measured by a flow cytometry assay. The percentage of apoptotic cells was significantly higher in IDH1-mu group than that in IDH1-wt and EV groups $(\mathrm{P}<0.05)$ (Fig. 4D). To investigate the migration and invasion ability of GSCs, we assayed the rate of cellular migration and invasion using Transwell assay system. The migrated and invasive cells in IDH1-mu group obviously decreased compared with other two groups $(\mathrm{P}<0.05)$ (Fig. 4E).

\section{Discussion}

Glioma is characterized by high morphological, genetic and phenotypic heterogeneity. However, its origin and the mechanisms which mediate its growth, recurrence and progression were still not fully understood (24). Studies have shown that the marked intratumoral heterogeneity, mirrored by the presence of distinct subpopulations of cells showing different tumorigenic capabilities, was one of the factors underlying tumor recurrence and poor long-term survival (26). In particular GSCs in glioma of different grades from both children and adults, a small subpopulation of cells with stem-like 
A

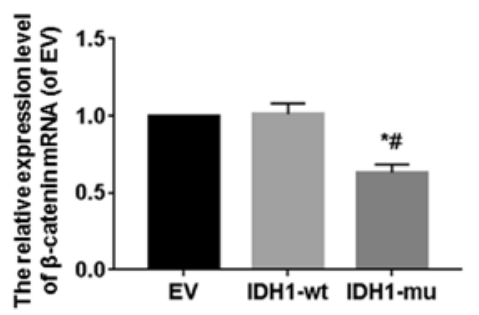

B
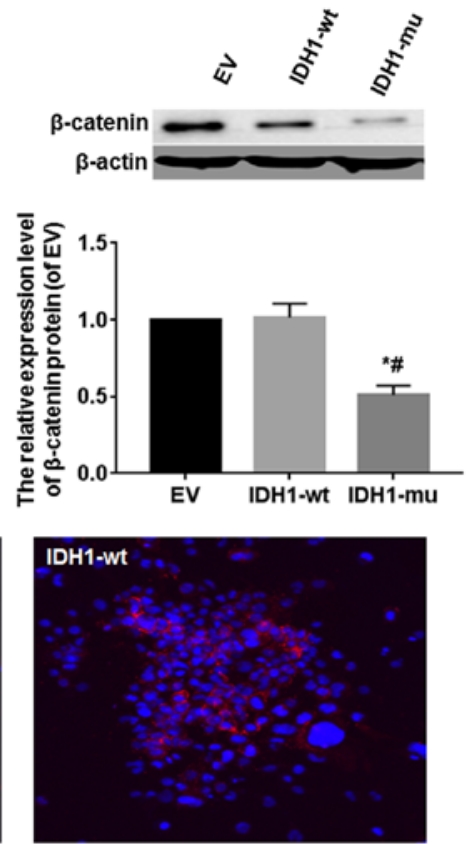

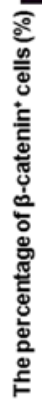

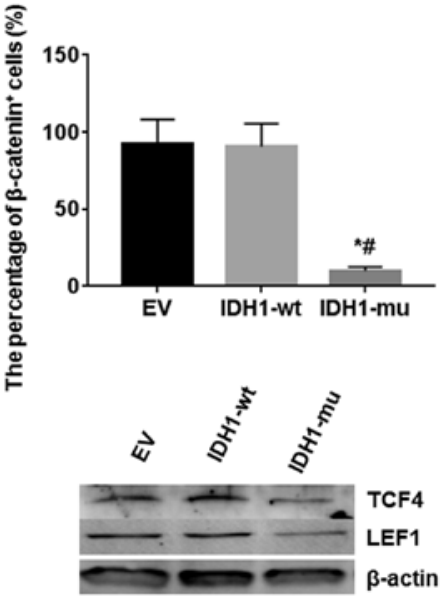

C

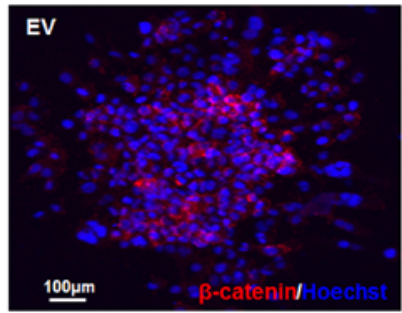

\section{IDH1-mu}
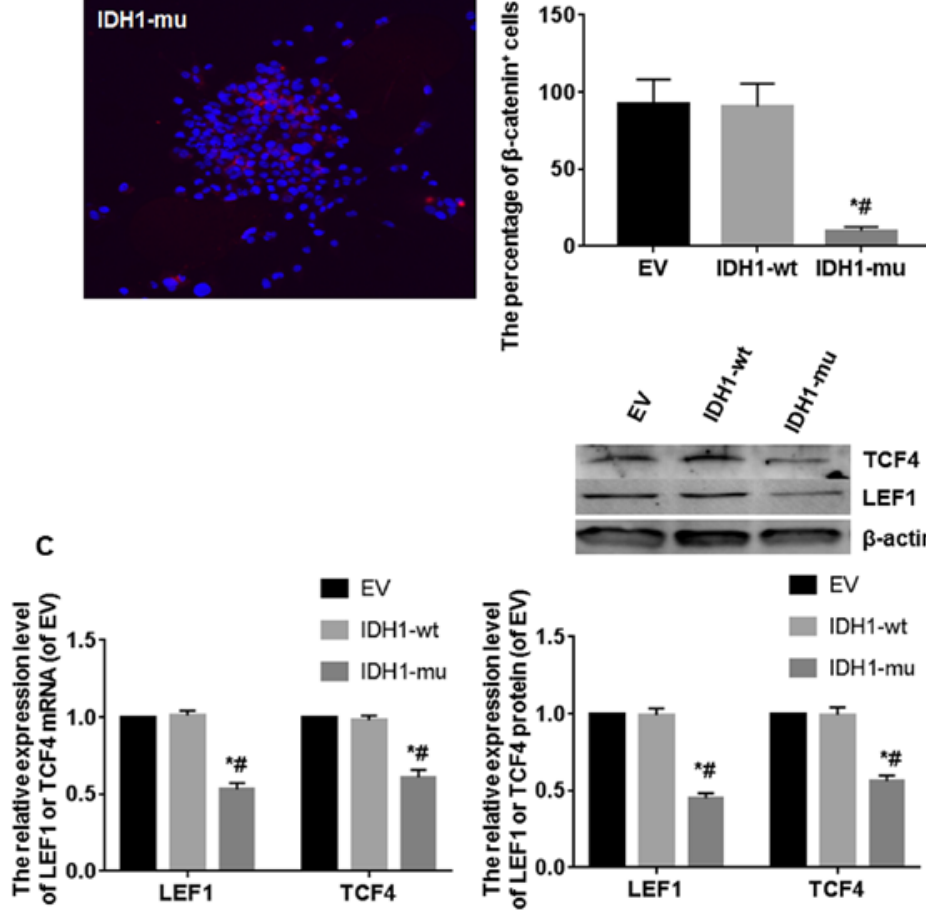

Figure 3. IDH1-R132H mutation reduced the activity of Wnt/ $\beta$-catenin signaling in GSCs. (A) $\beta$-catenin expression level was detected by RT-PCR and western blot analysis. Compared with IDH1-wt and EV group, the mRNA and protein expressions of $\beta$-catenin in IDH1-mu group significantly decreased. ${ }^{*} \mathrm{P}<0.05$ vs. EV; ${ }^{\prime} \mathrm{P}<0.05$ vs. IDH1-wt. (B) $\beta$-catenin expression level was detected by immunofluorescence. Many intense $\beta$-catenin ${ }^{+}$cells were found in the IDH1-mu group and few weak $\beta$-catenin ${ }^{+}$cells were found in the EV and IDH1-wt groups. Bar, $100 \mu \mathrm{m}$. ${ }^{*} \mathrm{P}<0.05$ vs. EV; ${ }^{*} \mathrm{P}<0.05$ vs. IDH1-wt. (C) The concentrations of $\beta$-catenin co-actors and Wnt signaling mediators TCF4 and LEF1 were measured by RT-PCR and western blot analysis. The mRNA and protein expression levels of TCF4 and LEF1 were downregulated in IDH1-mu group, compared with IDH1-wt and EV groups. ${ }^{*} \mathrm{P}<0.05$ vs. EV; ${ }^{\text {P }}<0.05$ vs. IDH1-wt.

properties, such as self-renewal capacity and a multilineage differentiation potential, are believed to be the real driving force for glioma initiation, progression and recurrence (5). GSCs give rise to a variety of proliferating and differentiated cells that make up the tumor mass and play a key role in the resistance to radiotherapy and chemotherapy $(7,8)$, angiogenesis (9) and metastasis (10). However, its molecular signal pathways are still unclear. As it has been shown in many studies, another feature of GSCs is the aberrant activation of several embryonic signaling pathways, such as Wnt signaling pathway to regulate self-renewal, migration and differentiation of GSCs (27-29).

Investigation of genetic mutations associated with glioma showed that patients frequently carried IDH1 mutations (12-14). Notably, the presence of the IDH1-R132H mutation significantly improved prognosis and longer progression-free and overall survival times in glioma patients $(14,30,31)$. Studies have shown that patients with IDH1-wt displayed a worse 
A
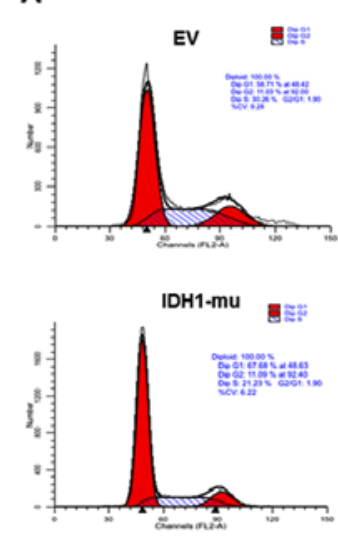

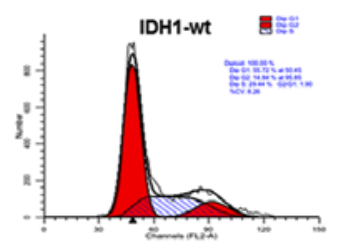

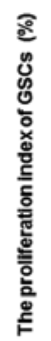

B
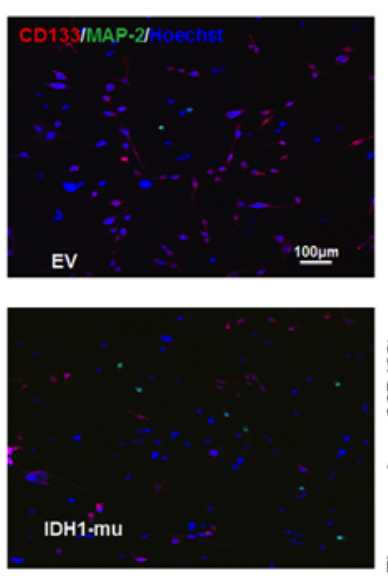
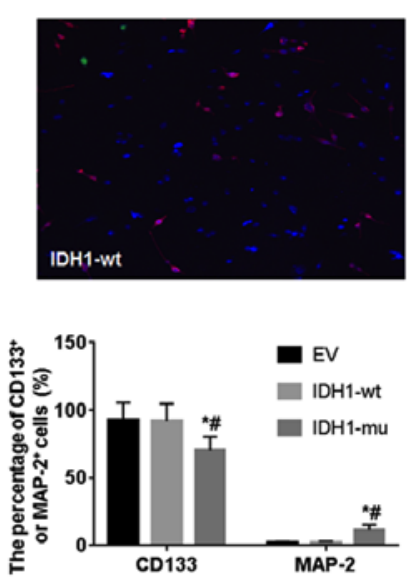

C

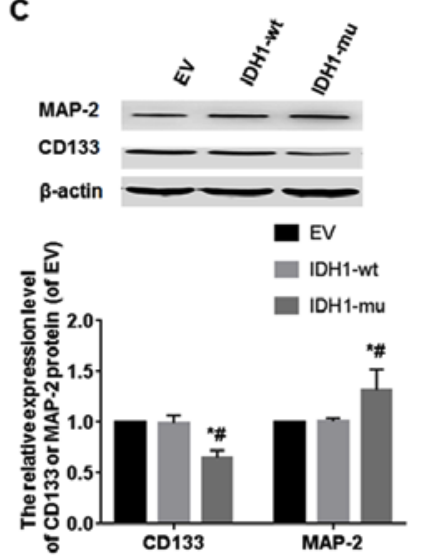

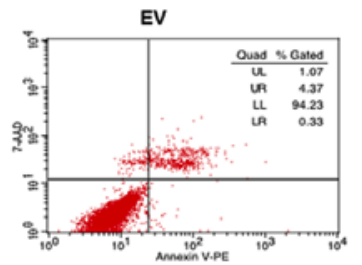
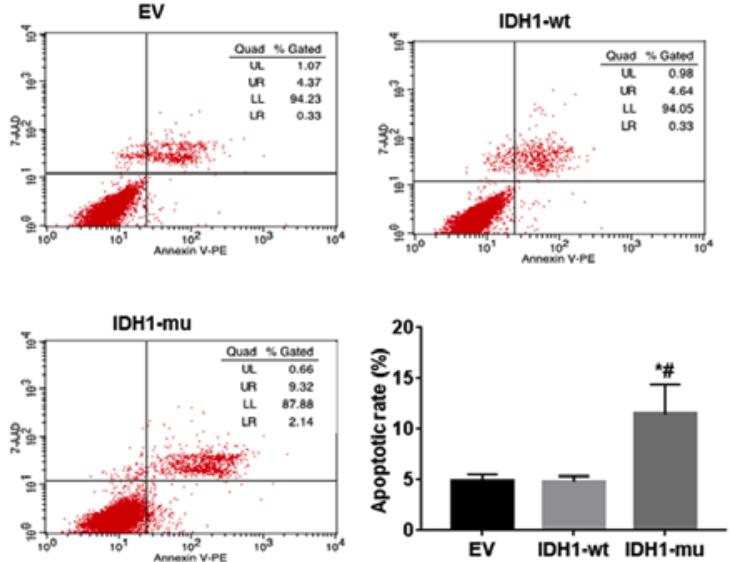

E
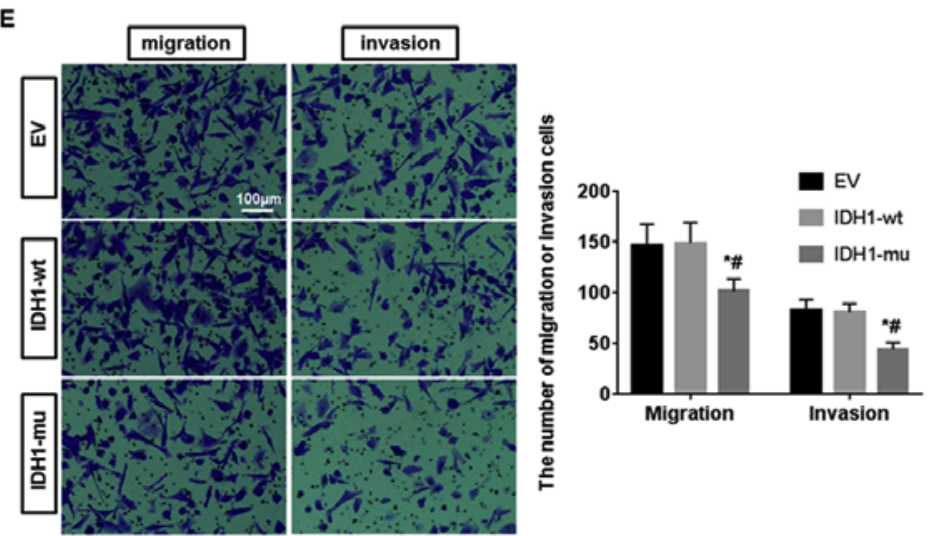

Figure 4. IDH1-R132H mutation led to a less aggressive phenotype in GSCs. (A) The proliferation ability of GSCs in 3 groups was detected by flow cytometry. The PI of IDH1-mu group was lower than that of the other two groups. " $\mathrm{P}<0.05$ vs. EV; ${ }^{\text {"P }}<0.05$ vs. IDH1-wt. (B) The differentiation ability of GSCs in 3 groups was detected by immunofluorescence. A few MAP- $2^{+}$neurons and many $\mathrm{CD} 133^{+}$cells were found in the 3 groups. Compared with IDH1-wt and EV group, the percentage of MAP- $2^{+}$neurons in IDH1-mu group was more than that of the other two groups, the percentage of CD133 $3^{+}$cells in IDH1-mu group was less than that of other two groups. Bar, $100 \mu \mathrm{m}$. ${ }^{*} \mathrm{P}<0.05$ vs. EV; ${ }^{\sharp} \mathrm{P}<0.05$ vs. IDH1-wt. (C) The CD133 and MAP-2 protein levels were detected by western blot analysis. Compared with IDH1-wt and EV groups, the CD133 protein level decreased and MAP-2 protein level increased in the IDH1-mu group. ${ }^{*} \mathrm{P}<0.05$ vs. EV; ${ }^{*} \mathrm{P}<0.05$ vs. IDH1-wt. (D) The apoptotic level of GSCs in 3 groups was measured by a flow cytometry assay. The percentage of apoptotic cells was significantly higher in IDH1-mu group than that in IDH1-wt and EV groups. ${ }^{*} \mathrm{P}<0.05$ vs. EV; ${ }^{\mathrm{P}} \mathrm{P}<0.05 \mathrm{vs}$. IDH1-wt. (E) The migration and invasion ability of GSCs were assayed using Transwell assay system. The migrated and invasive cells in IDH1-mu group obviously decreased compared with the other two groups. Bar, $100 \mu \mathrm{m}$. ${ }^{*} \mathrm{P}<0.05$ vs. EV; ${ }^{~} \mathrm{P}<0.05$ vs. IDH1-wt.

clinical outcome than those with IDH1-R132H. IDH1-R132H is now being used as a predictor of better prognosis in glioma patients (12). Our previous study showed that the $\mathrm{R} 132 \mathrm{H}$ mutation in IDH1 serves a tumor suppressor function in human glioma cells by negatively regulating $\mathrm{Wnt} / \beta$-catenin signaling (20). 
However, few studies have investigated the potential mechanistic cross-talk among IDH1 mutation, GSCs and Wnt/ $\beta$-catenin signaling. In the present study, we first showed an inverse correlation between the presence of the IDH1-R132H mutation and the $\mathrm{CD}_{133^{+}}$or nestin ${ }^{+}$cells in tumor samples obtained from glioma patients. CD133 (a cell surface marker for normal NSCs) and nestin (a cytoskeleton protein associated with NSCs and progenitor cells in CNS development) are recommended for the specific identification of GSCs (32-35). The results showed GSCs in the patients with IDH1-R132H were less than that in the patients with IDH1-wt. The positive rate of GSCs was significantly correlated with TNM stage and poor overall survival. To explore the relation between this mutation and GSCs, we overexpressed IDH1-R132H in GSCs isolated from patients with glioblastoma in vitro, as a control, we overexpressed either the wild-type IDH1 or the empty vector. A comprehensive examination of the cellular properties of the transfected GSCs in vitro showed that IDH1-R132H overexpression led to decreased proliferation, improved differentiation, inducing apoptosis and reducing the ability to migrate and invade surrounding substrate. At the molecular level, our data showed that the IDH1-R132H mutation triggered a significant reduction in the activity of Wnt/ $\beta$-catenin signaling. To confirm this conclusion, the expression of mediators, effectors and targets of canonical Wnt signaling, including $\beta$-catenin, TCF4 and LEF1 were downregulated in GSC overexpressing IDH1$\mathrm{R} 132 \mathrm{H}$, as determined by RT-PCR and western blot analysis.

In conclusion, the above data demonstrate that IDH1 mutation reduces the malignant progression of glioma by causing a less aggressive phenotype in GSCs which involve the Wnt/ $\beta$-catenin signaling. IDH1 may serve as a potential therapeutic target for glioma. However, further investigations are needed in order to deeply understand the biology of GSCs.

\section{Acknowledgements}

This study was supported by Jiangsu Provincial Key Medical Center, China Postdoctoral Science Foundation (grant no. 2015M581845), Postdoctoral Research Funding Plan in Jiangsu Province (grant no. 1601039A), Nantong Science and Technology Plan (grant no.MS32016016 and no. MS32015023), Young Medical Talent Project in Jiangsu Province (grant no. QNRC2016691).

\section{References}

1. Furnari FB, Fenton T, Bachoo RM, Mukasa A, Stommel JM, Stegh A, Hahn WC, Ligon KL, Louis DN, Brennan C, et al: Malignant astrocytic glioma: Genetics, biology, and paths to treatment. Genes Dev 21: 2683-2710, 2007.

2. Gladson CL, Prayson RA and Liu WM: The pathobiology of glioma tumors. Annu Rev Pathol 5: 33-50, 2010.

3. Stupp R, Mason WP, van den Bent MJ, Weller M, Fisher B, Taphoorn MJ, Belanger K, Brandes AA, Marosi C, Bogdahn U, et al; European Organisation for Research and Treatment of Cancer Brain Tumor and Radiotherapy Groups; National Cancer Institute of Canada Clinical Trials Group: Radiotherapy plus concomitant and adjuvant temozolomide for glioblastoma. $\mathrm{N}$ Engl J Med 352: 987-996, 2005.

4. Auffinger B, Tobias AL, Han Y, Lee G, Guo D, Dey M, Lesniak MS and Ahmed AU: Conversion of differentiated cancer cells into cancer stem-like cells in a glioblastoma model after primary chemotherapy. Cell Death Differ 21: 1119-1131, 2014.
5. Wen PY and Kesari S: Malignant gliomas in adults. N Engl J Med 359: 492-507, 2008.

6. Hemmati HD, Nakano I, Lazareff JA, Masterman-Smith M, Geschwind DH, Bronner-Fraser M and Kornblum HI: Cancerous stem cells can arise from pediatric brain tumors. Proc Natl Acad Sci USA 100: 15178-15183, 2003.

7. Beier D, Hau P, Proescholdt M, Lohmeier A, Wischhusen J, Oefner PJ, Aigner L, Brawanski A, Bogdahn U and Beier CP: $\mathrm{CD}_{133^{+}}$and CD133- glioblastoma-derived cancer stem cells show differential growth characteristics and molecular profiles. Cancer Res 67: 4010-4015, 2007.

8. Bao S, Wu Q, McLendon RE, Hao Y, Shi Q, Hjelmeland AB, Dewhirst MW, Bigner DD and Rich JN: Glioma stem cells promote radioresistance by preferential activation of the DNA damage response. Nature 444: 756-760, 2006.

9. Dean M, Fojo T and Bates S: Tumour stem cells and drug resistance. Nat Rev Cancer 5: 275-284, 2005.

10. Bao S, Wu Q, Sathornsumetee S, Hao Y, Li Z, Hjelmeland AB, Shi Q, McLendon RE, Bigner DD and Rich JN: Stem cell-like glioma cells promote tumor angiogenesis through vascular endothelial growth factor. Cancer Res 66: 7843-7848, 2006.

11. Dey M, Ulasov IV, Tyler MA, Sonabend AM and Lesniak MS: Cancer stem cells: The final frontier for glioma virotherapy. Stem Cell Rev 7: 119-129, 2011.

12. Uno M, Oba-Shinjo SM, Silva R, Miura F, Clara CA, Almeida JR, Malheiros SM, Bianco AM, Brandt R, Ribas GC, et al: IDH1 mutations in a Brazilian series of glioblastoma. Clinics (Sao Paulo) 66: 163-165, 2011.

13. Yan H, Parsons DW, Jin G, McLendon R, Rasheed BA, Yuan W, Kos I, Batinic-Haberle I, Jones S, Riggins GJ, et al: IDH1 and IDH2 mutations in gliomas. N Engl J Med 360: 765-773, 2009.

14. Yan W, Zhang W, You G, Bao Z, Wang Y, Liu Y, Kang C, You Y, Wang L and Jiang T: Correlation of IDH1 mutation with clinicopathologic factors and prognosis in primary glioblastoma: A report of 118 patients from China. PLoS One 7: e30339, 2012.

15. Parsons DW, Jones S, Zhang X, Lin JC, Leary RJ, Angenendt $P$, Mankoo P, Carter H, Siu IM, Gallia GL, et al: An integrated genomic analysis of human glioblastoma multiforme. Science 321: 1807-1812, 2008.

16. Sonoda Y, Kumabe T, Nakamura T, Saito R, Kanamori M, Yamashita Y, Suzuki H and Tominaga T: Analysis of IDH1 and IDH2 mutations in Japanese glioma patients. Cancer Sci 100: 1996-1998, 2009.

17. Dang L, White DW, Gross S, Bennett BD, Bittinger MA, Driggers EM, Fantin VR, Jang HG, Jin S, Keenan MC, et al: Cancer-associated IDH1 mutations produce 2-hydroxyglutarate. Nature 462: 739-744, 2009.

18. Xu W, Yang H, Liu Y, Yang Y, Wang P, Kim SH, Ito S, Yang C, Wang P, Xiao MT, et al: Oncometabolite 2-hydroxyglutarate is a competitive inhibitor of $\alpha$-ketoglutarate-dependent dioxygenases. Cancer Cell 19: 17-30, 2011.

19. Chitneni SK: IDH1 Mutations in Glioma: Considerations for radiotracer development. SM Radiol J 2: 1009, 2016.

20. Cui D, Ren J, Shi J, Feng L, Wang K, Zeng T, Jin Y and Gao L: $\mathrm{R} 132 \mathrm{H}$ mutation in IDH1 gene reduces proliferation, cell survival and invasion of human glioma by downregulating Wnt/ $\beta$-catenin signaling. Int J Biochem Cell Biol 73: 72-81, 2016.

21. Reya $\mathrm{T}$ and Clevers $\mathrm{H}$ : Wnt signalling in stem cells and cancer. Nature 434: 843-850, 2005.

22. Park JI, Venteicher AS, Hong JY, Choi J, Jun S, Shkreli M, Chang W, Meng Z, Cheung P, Ji H, et al: Telomerase modulates Wnt signalling by association with target gene chromatin. Nature 460: 66-72, 2009.

23. He H, Niu CS and Li MW: Correlation between glioblastoma stem-like cells and tumor vascularization. Oncol Rep 27: 45-50, 2012.

24. Li Q, Qiao G, Ma J and Li Y: Downregulation of VEGF expression attenuates malignant biological behavior of C6 glioma stem cells. Int J Oncol 44: 1581-1588, 2014.

25. Gurung PM, Veerakumarasivam A, Williamson M, Counsell N, Douglas J, Tan WS, Feber A, Crabb SJ, Short SC, Freeman A, et al: Loss of expression of the tumour suppressor gene AIMP3 predicts survival following radiotherapy in muscle-invasive bladder cancer. Int J Cancer 136: 709-720, 2015.

26. Dirks PB: Brain tumor stem cells: Bringing order to the chaos of brain cancer. J Clin Oncol 26: 2916-2924, 2008. 
27. Torres S, Lorente M, Rodríguez-Fornés F, Hernández-Tiedra S, Salazar M, García-Taboada E, Barcia J, Guzmán M and Velasco G: A combined preclinical therapy of cannabinoids and temozolomide against glioma. Mol Cancer Ther 10: 90-103, 2011.

28. Cheema TA, Kanai R, Kim GW, Wakimoto H, Passer B, Rabkin SD and Martuza RL: Enhanced antitumor efficacy of low-dose Etoposide with oncolytic herpes simplex virus in human glioblastoma stem cell xenografts. Clin Cancer Res 17: 7383-7393, 2011.

29. Costello JF, Berger MS, Huang HS and Cavenee WK: Silencing of $p 16 / C D K N 2$ expression in human gliomas by methylation and chromatin condensation. Cancer Res 56: 2405-2410, 1996.

30. Nobusawa S, Watanabe T, Kleihues P and Ohgaki H: IDH1 mutations as molecular signature and predictive factor of secondary glioblastomas. Clin Cancer Res 15: 6002-6007, 2009.
31. Xie F, Tang JJ, Wang X, Liu YH and Mao Q: Correlation between IDH1 mutation and prognosis in supratentorial high-grade astrocytomas. Sichuan Da Xue Xue Bao Yi Xue Ban 44: 184-187, 192 , 2013 (In Chinese).

32. Singh SK, Clarke ID, Terasaki M, Bonn VE, Hawkins C, Squire J and Dirks PB: Identification of a cancer stem cell in human brain tumors. Cancer Res 63: 5821-5828, 2003.

33. Singh SK, Hawkins C, Clarke ID, Squire JA, Bayani J, Hide T, Henkelman RM, Cusimano MD and Dirks PB: Identification of human brain tumour initiating cells. Nature 432: 396-401, 2004.

34. Shen G, Shen F, Shi Z, Liu W, Hu W, Zheng X, Wen L and Yang X: Identification of cancer stem-like cells in the C6 glioma cell line and the limitation of current identification methods. In Vitro Cell Dev Biol Anim 44: 280-289, 2008.

35. Zhou XD, Wang XY, Qu FJ, Zhong YH, Lu XD, Zhao P, Wang DH, Huang QB, Zhang L and Li XG: Detection of cancer stem cells from the C6 glioma cell line. J Int Med Res 37: 503-510, 2009. 